\title{
Factores Asociados al Dolor Musculoesquelético en Población Trabajadora Chilena
}

\author{
FACTORS ASSOCIATED WITH MUSCULOSKELETAL PAIN IN CHILEAN WORKING POPULATION
}

Carolina Vidal Gamboa', Lorena Hoffmeister Arce', Dafna Benadof'

1. Escuela de Salud Pública, Universidad Mayor, Santiago, Chile.

\begin{abstract}
RESUMEN
Objetivo: Identificar factores asociados al reporte de dolor atribuido al trabajo en la población chilena. Métodos: Se realizó un análisis utilizando la Primera Encuesta Nacional de Condiciones de Empleo, Trabajo, Salud y Calidad de Vida de trabajadores en Chile 20092010. La variable resultado fue el número de localizaciones con reporte de dolor atribuido al trabajo. Se incluyeron en el análisis factores demográficos y socioeconómicos, condiciones de trabajo, conductas de salud y factores personales. Se utilizó regresiones de Poisson cero-inflado para estimar la contribución de los distintos factores sobre el dolor reportado. Resultados: Uno de cada dos trabajadores reporta dolor. Ser mujer se asocia a un mayor reporte de dolor (RP: 1,47), disminuyendo en trabajadoras de edad avanzada (RP: 1,37). Los trabajadores expuestos a demandas físicas reportan 64\% más localizaciones con dolor. La demanda psicológica, el bajo apoyo socio-laboral y la baja autonomía en el trabajo se asocian a mayor reporte de dolor (RP: 1,29, RP: 1,13, RP: 1,23, respectivamente). Discusión y Conclusiones: El género y la edad son factores relevantes en el reporte del dolor asociado a trabajo. Este efecto es independiente de las distintas condiciones laborales, identificando importantes diferencias entre los distintos grupos de edad.
\end{abstract}

(Vidal C, Hoffmeister L, Benadof D, 2016. Factores Asociados al Dolor Musculoesquelético en Población Trabajadora Chilena. Un Estudio de Caso. Cienc Trab. Ene-Abr; 18 [55]: 23-27).

Palabras claves: DOLOR MUSCULOESQUELÉTICO, CONDICIONES DE TRABAJO, CLASE SOCIAL, MODELOS CERO INFLADO, CHILE.

\section{ABSTRACT}

Objetive: Identify factors associated to pain due to work related activities, reported by chilean workers.

Methods: We conducted an analysis of the First National Survey of Work Conditions, Work, Health and workers' Quality of Life, conducted in Chile in 2009-2010. The result variable was the reported number of body parts with pain due to work. The analysis included sociodemographic variables, working conditions, health behaviors, and personal variables. We conducted zero inflated Poisson regressions to estimate the contribution of the factors on reported pain. Results: One of every two workers reported pain. Women reported more pain than men (PR: 1.47), but this relationship diminished as age increased (PR: 1.37). Workers that were exposed to physical demands reported pain in 64\% more body parts. Psychological demand, low social support in work, and low work autonomy were associated to more pain report (PR: 1.29, PR: 1.13, PR: 1.23 respectively).

Conclusions: Gender and age are relevant factors associated to pain report due to work, even when adjusted for working conditions. Significant differences between age groups were identified.

Key words: MUSCULOSKELETAL PAIN, WORKING CONDITIONS, SOCIAL CLASS, ZERO INFLATED MODELS, CHILE.

\section{INTRODUCCIÓN}

El dolor crónico es un problema importante en la comunidad, afectando la salud general, salud mental y bienestar social y económico. ${ }^{1-3} \mathrm{El}$ dolor es una de las causas que más motivan las consultas médicas. ${ }^{2}$ Los trastornos musculoesqueléticos representan un importante problema para la salud pública, no sólo por su alta prevalencia e incidencia sino también debido al alto impacto en la funcionalidad de las personas, en las discapacidades laborales, y en los altos costos económicos asociados al uso de

Correspondencia / Correspondence:

Dafna Benadof

José Toribio Medina 38, Santiago, Chile

Tel.: (56) 223281788

e-mail: dafna.benadof@umayor.cl

Recibido: 11 de Noviembre de 2015 / Aceptado: 26 de Enero 2016

servicios de salud, ausentismo laboral y retiro prematuro. ${ }^{4}$ Los pacientes que presentan dolor crónico usan los servicios cinco veces más que el resto de la población. ${ }^{2,5}$

En países occidentales, los trastornos dolorosos de espalda, cuello y extremidades superiores son las principales causas de discapacidad en el trabajo. ${ }^{6}$ Se estima que aproximadamente uno de cada cinco personas de la población adulta en Europa padece dolor crónico. ${ }^{7} \mathrm{El}$ costo asociado al dolor es considerable, siendo por sobre $€ 200 \mathrm{mil}$ millones por año en Europa, y \$635 mil millones por año en USA en el 2008. ${ }^{8}$ En Chile, Hoffmeister et al. ${ }^{9}$ reportaron el año 2012 que un tercio de los trabajadores presentaba dolores permanentes o recurrentes en la espalda y un $28,2 \%$ en la zona lumbar.

Las exposiciones físicas, particularmente la manipulación de materiales pesados, la realización de movimientos repetitivos, la exposición a posturas forzadas y a vibraciones están asociadas al dolor musculoesquelético. ${ }^{10}$ Otros estudios han encontrado una asociación entre factores psicológicos, tales como las tendencias somatizantes y el estado de ánimo y la declaración de dolor. ${ }^{11,12}$ Estudios más recientes han investigado el efecto de factores psicosociales, 
tales como el control y la demanda del trabajo ${ }^{13-14}$, sobre el dolor atribuido al trabajo.

La prevalencia de dolor es más alta en las personas de edad avanzada que en los más jóvenes, y por género es mayor en mujeres que en hombres. ${ }^{15}$ Existe una gran variabilidad en la prevalencia de dolor entre países europeos. Según Farioli et al. ${ }^{16}$ los factores de riesgo a nivel individual no pueden explicar toda esta variabilidad, evidenciando el rol de otros factores como los socioeconómicos. Diversos estudios muestran de manera consistente que la prevalencia de dolor se asocia de manera inversa al nivel socioeconómico ${ }^{17,18}$, siendo más severo en grupos menos privilegiados. ${ }^{19}$

El objetivo del presente estudio es identificar factores relacionados con el reporte de dolor atribuido al trabajo en la población chilena, para distintos grupos de edad.

\section{MATERIALES Y MÉTODOS}

Se realizó un análisis secundario de datos de la Primera Encuesta Nacional de Condiciones de Empleo, Trabajo, Salud y Calidad de Vida de los trabajadores y trabajadoras en Chile (ENETS 20092010). ${ }^{20}$ Esta encuesta utilizó un diseño muestral probabilístico, multietápico y estratificado geográficamente. La muestra fue de 9.503 personas entrevistadas en sus hogares, representando a la población trabajadora de Chile de 15 o más años de edad.

\section{Variables de estudio}

La variable de resultado fue el número de localizaciones con presencia de dolor provocado por trabajo. Esta variable fue construida a través de dos preguntas del cuestionario: a) "En los últimos 12 meses, ¿Ud. ha tenido dolores permanentes o recurrentes en algunas de las siguientes partes del cuerpo?” (14 localizaciones), y b) “¿Este dolor es, en alguna medida, provocado por el trabajo que realiza?”. Se creó una variable continua, contando las afirmaciones de presencia de dolor provocado por el trabajo, por lo cual la variable presenta valores de 0 a 14, donde 0 corresponde a ninguna localización con dolor y 14 corresponde a declaración de dolor en las 14 localizaciones.

Entre los factores demográficos y socioeconómicos se incluyó: género, edad, ingresos mensuales debido al trabajo, situación laboral actual (empleado/desempleado) y clase social. Para esta última variable se utilizó una adaptación de la perspectiva de clase social neomarxista basado en el modelo de Wright. ${ }^{21,22}$ Dicho esquema contiene 12 categorias o posiciones de clase distribuidas en tres dimensiones: relaciones de propiedad, control sobre la organización del trabajo y credenciales/habilidades. Esta construcción se sustentó en la selección de preguntas realizada por Rocha. ${ }^{23}$

También se incorporaron dimensiones referidas a condiciones de trabajo, incluyéndose: a) exposición a demandas físicas: estar expuesto toda o la mitad de la jornada a posturas incómodas, trasladar o mover cargas pesadas o a realización de movimientos repetitivos en cortos períodos de tiempo; b) exposición a demandas psicosociales del trabajo: nunca o rara vez su jornada de trabajo le alcanza para mantener al día sus tareas, también se consideraron aquellos trabajadores que siempre o casi siempre deben resolver problemas difíciles o su trabajo le exige gran esfuerzo de concentración y/o atención; c) exposición a bajo control y autonomía: nunca o rara vez pueden influir en la cantidad de trabajo que le asignan, cambiar el orden de sus tareas, o cambiar la velocidad o el método con que debe trabajar; d) exposición a bajo apoyo socio-laboral: nunca o rara vez sus compañeros o jefes escuchan los problemas asociados al trabajo.
Se incluyeron tres conductas relacionadas con la salud: a) actividad física recomendada: realizar actividad física durante 30 minutos o más, 3 o más veces por semana; b) consumo actual de tabaco: fumar al menos un cigarrillo durante este último mes; y c) bebedor problema: según la escala EBBA. ${ }^{24}$ Finalmente, se incorporaron factores personales referidos a la percepción de distintos aspectos de la vida personal y laboral, incluyéndose: ¿Cómo se siente usted con la vida familiar?, ¿Cómo se siente usted con su nivel de endeudamiento?, para estos dos factores se consideró expuestos aquellos que reportaron una percepción menos que buena. Otro factor fue, ¿Le deja el trabajo suficiente tiempo libre para hacer otras cosas que desea hacer? Se consideró expuestos a los trabajadores que contestaron nunca o rara vez.

\section{Análisis de datos}

Se realizó un análisis descriptivo para estimar la prevalencia de al menos una localización con reporte de dolor, utilizando proporciones e intervalos de confianza del 95\%. Para la variable de resultado se calculó el valor medio del número de localizaciones con reporte de dolor atribuido al trabajo junto con sus intervalos de confianza de 95\%. Para determinar la relación existente entre el reporte de dolor atribuido al trabajo y los distintos factores se utilizó la regresión de Poisson y binomial negativo. ${ }^{25}$ Éstos son modelos para datos de conteo. Debido a que la población trabajadora es considerada sana, se obtuvo una alta proporción de trabajadores sin reporte de dolor, por lo que se contrastó el uso de modelos cero inflado ${ }^{26}$ : Poisson cero inflado (ZIP por sus siglas en inglés) y binomial negativo cero inflado (ZINB por sus siglas en inglés). La comparación de modelos se realizó mediante el test de Vuong ${ }^{26,27}$, el cual compara el modelo cero inflado con un modelo de conteo estándar. En éste, una prueba significativa (p-valor $<0,05$ ) indica que el modelo cero inflado es mejor que el estándar. Se utilizó Likelihood-ratio tes $\mathrm{t}^{27}$ para comparar el modelo ZINB con el modelo ZIP, donde una prueba significativa (p-valor < $0,05)$ indica el ZINB como mejor modelo. El ajuste de modelos cuenta con estimaciones robustas de la varianza como lo recomiendan Cameron \& Trivedi. ${ }^{28}$ Se calcularon cuatro modelos: uno para la población trabajadora general, y un modelo para cada grupo de edad, de 15 a 30 años (trabajadores jóvenes), de 31 a 59 años (trabajadores de edad media), de 60 años y más (trabajadores de edad avanzada). Para todos los modelos se incorporaron los factores antes descritos. Los análisis fueron realizados utilizando STATA 13.

\section{RESULTADOS}

La población de estudio está constituida por un 59,9\% de hombres. Un $87,9 \%$ estaba empleado al momento de la encuesta. Un $67,1 \%$ de los trabajadores declaró estar expuesto a demandas psicológicas. Además, un 52,1\% tenía una percepción menos que buena respecto a su nivel de endeudamiento (Tabla 1).

Un 49,8\% [IC95\%: 47,5\%-52,1\%] de los trabajadores reportó dolor en al menos una localización. El promedio de localizaciones con reporte de dolor fue de 1,7 [IC95\%: 1,5-1,8]. Un 50,2\% [IC95\%: 47,9\%-52,5\%] de los trabajadores declararon no tener dolor atribuible al trabajo (Figura 1).

Los resultados de los modelos de regresión, tanto para el conjunto de la población trabajadora como para cada grupo de edad, son presentados en la Tabla 2. Para la estimación de los coeficientes, se eligió el modelo binomial negativo cero inflado (Test de Voung, $\operatorname{Pr}>\mathrm{z}=0,00$; Likelihood-ratio test, $\operatorname{Pr}>=$ chibar2 =0,00). Para el 
Tabla 1.

Descripción de la población de estudio y de las principales variables de estudio.

\begin{tabular}{|c|c|c|}
\hline & $\mathrm{N}$ & \% e IC(95) \\
\hline \multicolumn{3}{|l|}{ Factores demográficos y socioeconómicos } \\
\hline \multicolumn{3}{|l|}{ Género } \\
\hline Hombre & 5.958 & $59,9 \%(57,6 \%-62,2 \%)$ \\
\hline Mujer & 3.545 & $40,1 \%(37,8 \%-42,4 \%)$ \\
\hline \multicolumn{3}{|l|}{ Grupos de edad } \\
\hline 15 a 30 años & 1.954 & $25,0 \%(23,0 \%-27,0 \%)$ \\
\hline 31 a 59 años & 6.367 & $63,8 \%(61,6 \%-66,0 \%)$ \\
\hline 60 y más años & 1.182 & $11,2 \%(9,8 \%-12,8 \%)$ \\
\hline \multicolumn{3}{|l|}{ Situación laboral } \\
\hline Empleado & 8.356 & $87,9 \%(86,6 \%-89,2 \%)$ \\
\hline Desempleado & 1.147 & $12,1 \%(10,8 \%-13,4 \%)$ \\
\hline \multicolumn{3}{|l|}{ Ingresos mensuales del trabajo } \\
\hline Menos de $\$ 136.000$ & 2.047 & $22,1 \%(20,0 \%-24,4 \%)$ \\
\hline$\$ 137.000-\$ 180.000$ & 2.229 & $22,2 \%(20,5 \%-24,0 \%)$ \\
\hline$\$ 181.000-\$ 250.000$ & 1.778 & $18,9 \%(17,3 \%-20,6 \%)$ \\
\hline$\$ 251.000-\$ 450.000$ & 2.088 & $23,5 \%(21,6 \%-25,5 \%)$ \\
\hline$\$ 451.000-\$ 850.000$ & 806 & $9,4 \%(8,0 \%-11,1 \%)$ \\
\hline$\$ 851.000$ y más & 300 & $3,9 \%(2,9 \%-5,2 \%)$ \\
\hline \multicolumn{3}{|l|}{ Condiciones de trabajo } \\
\hline Expuesto a demandas físicas & 4.097 & $41,7 \%(39,6 \%-44,0 \%)$ \\
\hline Expuesto a demandas psicológicas & 6.281 & $67,1 \%(64,9 \%-69,2 \%)$ \\
\hline Bajo control y autonomía & 5.672 & $61,4 \%(59,2 \%-63,6 \%)$ \\
\hline Bajo apoyo socio-laboral & 2.383 & $27,0 \%(24,9 \%-29,2 \%)$ \\
\hline \multicolumn{3}{|l|}{ Conductas relacionadas con la salud } \\
\hline Actividad fisica recomendada & 598 & $6,5 \%(5,5 \%-7,6 \%)$ \\
\hline Fumador actual & 3.588 & $38,0 \%(35,8 \%-40,3 \%)$ \\
\hline Bebedor problema & 456 & $4,5 \%(3,8 \%-5,3 \%)$ \\
\hline \multicolumn{3}{|l|}{ Factores personales } \\
\hline Percepción de la vida familiar: menos que buena & 1.418 & $17,5 \%(15,6 \%-19,7 \%)$ \\
\hline Percepción del endeudamiento: menos que buena & 4.673 & $52,1 \%(49,8 \%-54,4 \%)$ \\
\hline Percepción del tiempo libre: menos que buena & 3.803 & $40,3 \%(38,1 \%-42,5 \%)$ \\
\hline
\end{tabular}

desarrollo de los modelos se excluyó del análisis un 5,8\% de los datos (548 casos) debido a falta de información asociada a las variables utilizadas en el estudio.

Para el conjunto de la población trabajadora, los factores que contribuyen significativamente al reporte de dolor son: género, ingresos del trabajo y condiciones de trabajo. Ser mujer se asocia a un mayor reporte de dolor (RP: 1,47) en comparación con los hombres. Con

Figura 1.

Histograma del número de localizaciones con declaración de dolor.

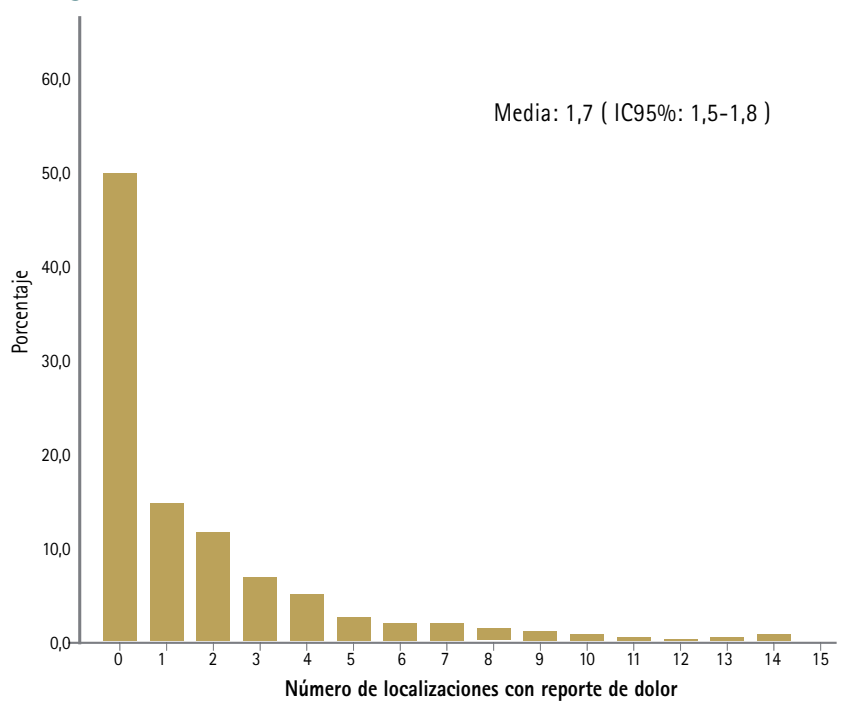

respecto a los ingresos del trabajo, los trabajadores con menores ingresos presentan una mayor probabilidad de reportar dolor, y los trabajadores que reciben un ingreso mensual menor de $\$ 136.000$ (\$194 US) presentan un reporte de dolor 68\% mayor que los trabajadores que reciben mensualmente $\$ 851.000$ (\$1214 US) o más. Los trabajadores expuestos a demandas físicas tienen 64\% más localizaciones con dolor que sus pares no expuestos. La demanda psicológica, el bajo apoyo socio-laboral y la baja autonomía en el trabajo también se asocian a un mayor reporte de dolor. Los trabajadores que se identificaron como "bebedores problemas" presentan un reporte 57\% mayor que el resto de los trabajadores. El empresariado, la pequeña burguesía y los gerentes básicos tienen una probabilidad mayor de reporte de dolor que trabajadores clasificados como proletariado informal. Se encontró que todos los factores personales tienen una contribución significativa, con un aumento del reporte del dolor cuando la percepción personal es menos que buena.

Los modelos por grupo de edad muestran que el hecho de ser mujer se mantiene como un factor estadísticamente significativo a lo largo de los distintos grupos de edad. Sin embargo, la magnitud del coeficiente disminuye a medida que se pasa a una categoría de edad superior. El efecto de los ingresos del trabajo difiere según el grupo edad al que pertenece el trabajador. Los trabajadores de edad media que reciben un ingreso mensual menor a \$136.000 (\$194 US) reportan un 70\% más de localizaciones con dolor comparado con aquellos trabajadores con un ingreso de $\$ 851.000$ (\$1214 US) o más. Éste coeficiente disminuye a medida que el ingreso por trabajo aumenta. En los trabajadores de 60 años y más, aquellos con menor ingreso por trabajo, reportan 2,8 veces mayor de dolor en comparación con trabajadores del mismo grupo etario que presentan mayores ingresos. El efecto, independiente de la clase social, no es significativo en los modelos separados por grupo de edad. La exposición a demandas físicas tiene un RP de 1,98 entre los trabajadores jóvenes, siendo de 1,63 entre los trabajadores de edad media y de 1,32 en los de edad avanzada. La exposición a demandas psicológicas y el bajo apoyo socio-laboral no son factores que contribuyan significativamente al reporte de dolor entre los trabajadores de edad avanzada, a diferencia de lo que acontece para los grupos de menor edad. El bajo control y autonomía laboral aumenta el reporte de dolor en los trabajadores de edad media (RP 1,14 [IC 95\%] 1,04-1,23] y avanzada (RP 1,34 [IC 95\% 1,10-1,63], y la contribución no es significativa para los trabajadores jóvenes. Por otro lado, la baja satisfacción con el nivel de endeudamiento y la percepción de tiempo libre menos que buena, aumentan el reporte de dolor en los trabajadores jóvenes y de edad media, no así en los trabajadores de edad avanzada.

\section{DISCUSIÓN}

Encontramos una alta prevalencia de declaración de dolor asociada al trabajo. Uno de cada dos trabajadores reporta al menos una localización con dolor atribuido al trabajo. Esta proporción es menor a la reportada por Reindhart et $\mathrm{al}^{29}$, quien encontró un 75\% en población general de Suiza.

El análisis multivariado evidencia que ser mujer es una de las condiciones que más contribuye al reporte de dolor, incluso ajustando por variables como clase social e ingresos por trabajo. Esto podría ser atribuido a la segmentación del mercado laboral, esto es, mujeres y hombres difieren en su situación laboral, tipo de ocupación, jerarquía en la empresa y en las tareas asignadas. ${ }^{30,31}$ En esta dirección, estudios realizados en Chile han mostrado que la condición de género 
Tabla 2.

Modelo de regresión binomial negativo cero-inflado para asociación entre factores demográficos, socioeconómicos, condiciones de trabajo y conductas de salud, y el número de localizaciones corporales con reporte de dolor atribuido al trabajo.

\begin{tabular}{|c|c|c|c|c|}
\hline & $\begin{array}{l}\text { Modelo Población } \\
15 \text { y más años } \\
\text { RP (IC95\%) }\end{array}$ & $\begin{array}{l}\text { Trabajadores } \\
\text { Jóvenes } \\
\text { RP (IC95\%) }\end{array}$ & $\begin{array}{l}\text { Trabajadores } \\
\text { Edad media } \\
\text { RP (IC95\%) }\end{array}$ & $\begin{array}{l}\text { Trabajadores } \\
\text { Edad avanzada } \\
\text { RP (IC95\%) }\end{array}$ \\
\hline \multicolumn{5}{|l|}{ Factores demográficos y socioeconómicos } \\
\hline Género: $:$ ujer $^{a}$ & $1,47(1,46-1,57)$ & $1,61(1,37-1,92)$ & $1,51(1,39-1,64)$ & $1,37(1,09-1,71)$ \\
\hline \multicolumn{5}{|l|}{ Clase social } \\
\hline Empresariado & $1,35(0,99-1,82)$ & $0,89(0,48-1,67)$ & $1,41(0,94-2,12)$ & $1,31(0,57-3,01)$ \\
\hline Mediano Empresariado & $1,13(0,94-1,35)$ & $0,91(0,89-1,67)$ & $1,11(0,88-1,41)$ & $1,09(0,66-1,82)$ \\
\hline Pequeña burguesia & $1,20(1,03-1,39)$ & $0,69(0,41-1,16)$ & $1,13(0,93-1,38)$ & $1,29(0,78-2,12)$ \\
\hline Gerente expertos & $0,82(0,63-1,06)$ & $0,56(0,27-1,18)$ & $0,79(0,58-1,08)$ & $0,84(0,46-1,52)$ \\
\hline Supervisor experto & $1,15(0,87-1,52)$ & $1,10(0,53-2,32)$ & $1,01(0,74-1,39)$ & $2,00(0,99-4,01)$ \\
\hline Obrero calificado & $0,98(0,82-1,18)$ & $0,74(0,46-1,17)$ & $1,00(0,80-1,25)$ & $1,07(0,44-2,63)$ \\
\hline Gerente semicalificados & $1,12(0,81-1,55)$ & $1,16(0,60-2,24)$ & $1,15(0,80-1,64)$ & $0,41(0,09-1,82)$ \\
\hline Supervisor semicalificado & $1,03(0,83-1,29)$ & $1,13(0,68-1,87)$ & $0,95(0,73-1,25)$ & $1,19(0,37-3,88)$ \\
\hline Obrero semicalificado & $0,89(0,78-1,03)$ & $0,85(0,58-1,24)$ & $0,91(0,76-1,10)$ & $0,94(0,58-1,54)$ \\
\hline Gerente básico & $1,33(1,03-1,72)$ & $1,99(0,83-4,78)$ & $1,22(0,90-1,66)$ & $1,60(0,67-3,78)$ \\
\hline Supervisor básico & $0,96(0,72-1,31)$ & $1,09(0,58-2,05)$ & $0,86(0,62-1,20)$ & $1,26(0,44-3,57)$ \\
\hline Proletariado Formal & $1,10(0,94-1,31)$ & $0,82(0,50-1,36)$ & $1,09(0,88-1,33)$ & $1,27(0,76-2,12)$ \\
\hline Proletariado Informal & 1 & 1 & 1 & 1 \\
\hline Situación laboral: Empleado ${ }^{b}$ & $1,50(1,32-1,69)$ & $1,85(1,40-2,48)$ & $1,40(1,19-1,66)$ & $1,18(0,81-1,71)$ \\
\hline \multicolumn{5}{|l|}{ Ingresos } \\
\hline Menos de $\$ 136,000$ & $1,68(1,35-2,08)$ & $0,54(0,26-1,12)$ & $1,70(1,24-2,25)$ & $2,82(1,27-6,31)$ \\
\hline$\$ 137,000-\$ 180,000$ & $1,48(1,20-1,82)$ & $0,60(0,30-1,24)$ & $1,50(1,12-2,00)$ & $2,14(0,97-4,70)$ \\
\hline$\$ 181,000-\$ 250,000$ & $1,45(1,17-1,80)$ & $0,66(0,32-1,34)$ & $1,47(1,10-1,97)$ & $2,05(0,92-4,57)$ \\
\hline$\$ 251,000-\$ 450,000$ & $1,52(1,24-1,87)$ & $0,64(0,31-1,30)$ & $1,58(1,19-2,10)$ & $1,88(0,85-4,19)$ \\
\hline$\$ 451,000-\$ 850,000$ & $1,32(1,06-1,65)$ & $0,69(0,33-1,47)$ & $1,30(0,97-1,74)$ & $2,20(0,98-4,93)$ \\
\hline$\$ 851,000$ y más & 1 & 1 & 1 & 1 \\
\hline \multicolumn{5}{|l|}{ Condiciones de trabajo ${ }^{c}$} \\
\hline Expuesto a demandas físicas & $1,64(1,54-1,76)$ & $1,98(1,67-2,35)$ & $1,63(1,50-1,77)$ & $1,32(1,08-1,61)$ \\
\hline Expuesto a demandas psicológicas & $1,29(1,20-1,39)$ & $1,27(1,05-1,54)$ & $1,33(1,22-1,46)$ & $1,15(0,95-1,38)$ \\
\hline Bajo control y autonomía & $1,13(1,05-1,22)$ & $0,93(0,76-1,18)$ & $1,14(1,04-1,23)$ & $1,34(1,10-1,63)$ \\
\hline Bajo apoyo socio-laboral & $1,23(1,14-1,33)$ & $1,35(1,13-1,61$ & $1,21(1,11-1,32)$ & $1,19(0,93-1,52)$ \\
\hline \multicolumn{5}{|l|}{ Conductas de salud } \\
\hline Actividad fisica recomendada ${ }^{d}$ & $1,01(0,89-1,15)$ & $1,17(0,91-1,52)$ & $1,03(0,88-1,22)$ & $0,85(0,51-1,43)$ \\
\hline Fumador actual $^{\mathrm{e}}$ & $0,99(0,92-1,05)$ & $0,95(0,80-1,12)$ & $0,92(0,85-0,99)$ & $1,20(0,92-1,56)$ \\
\hline Bebedor problema ${ }^{f}$ & $1,57(1,36-1,82)$ & $1,85(1,31-2,62)$ & $1,40(1,19-1,65)$ & $2,18(1,43-3,34)$ \\
\hline \multicolumn{5}{|l|}{ Factores personales $^{g}$} \\
\hline Percepción de la vida familiar: menos que buena & $1,19(1,08-1,30)$ & $1,21(0,98-1,50)$ & $1,19(1,08-1,32)$ & $1,05(0,83-1,34)$ \\
\hline Percepción del endeudamiento: menos que buena & $1,20(1,11-1,28)$ & $1,18(1,01-1,39)$ & $1,20(1,11-1,30)$ & $1,11(0,92-1,32)$ \\
\hline Percepción del tiempo libre: menos que buena & $1,32(1,28-1,41)$ & $1,28(1,08-1,50)$ & $1,34(1,24-1,45)$ & $1,19(0,99-1,44)$ \\
\hline
\end{tabular}

${ }^{a}$ Referencia: Hombre, ${ }^{b}$ Referencia: Desempleado, ${ }^{c}$ No expuesto a las condiciones de trabajo, ${ }^{d}$ Baja actividad física, ${ }^{e}$ No fumador, ${ }^{f}$ No presenta problemas de alcohol.

tiene una asociación clara con las condiciones de empleo de hombres y mujeres. ${ }^{32}$ Asimismo, las mujeres trabajadoras en Chile se encuentran en posiciones menos ventajosas que los hombres con respecto a su trabajo, especialmente en lo referido al ingreso por trabajo, donde las mujeres a pesar de tener el mismo nivel educacional que los hombres, presentan un nivel de ingresos menor. ${ }^{32}$ Otros estudios señalan que una mayor prevalencia de dolor musculoesquelético entre mujeres que trabajan puede estar vinculado al hecho de que las mujeres son responsables de hacer la mayor parte de las tareas del hogar. ${ }^{33}$ El análisis multivariado también nos mostró que los trabajadores clasificados en pequeña burguesía y en gerente básico tienen una mayor probabilidad de reportar dolor comparado con el grupo de proletariado informal. Esto concuerda con lo encontrado por Rocha et $\mathrm{al}^{23}$, quienes describieron mayor prevalencia de mala salud percibida en el grupo de pequeña burguesía. Otras evidencias indican que la vinculación entre relaciones de empleo y salud no es necesariamente gradual, con pequeños empleadores o supervisores presentando peores resultados que otros trabajadores. ${ }^{34}$

En el conjunto de la población trabajadora encontramos que ser bebedor problema se asocia de manera significativa con la declaración de dolor. Estudios muestran que los efectos del alcohol sobre el dolor crónico se producen cuando éste se consume en forma excesiva para la salud, y el retiro del uso crónico de alcohol a menudo aumenta la sensibilidad al dolor. ${ }^{35}$ Nuestros resultados muestran que la asociación se mantiene para todos los grupos de edad, pero con una mayor magnitud entre los trabajadores de edad avanzada, con un RP de 2,18 .

El bajo control y autonomía del trabajo se asocia al reporte de dolor. Estudios muestran que la influencia de un bajo control de trabajo es un factor de riesgo ocupacional para la aparición o persistencia de dolor. ${ }^{14}$ Para el grupo de trabajadores jóvenes no se observa una asociación significativa, pudiendo deberse que este grupo es el que tiene un menor tiempo en el mercado laboral.

Las fortalezas del estudio corresponden a que la información proviene de una muestra representativa de la población trabajadora de Chile. Además se incluyó como factor la clase social diferenciando el proletariado informal del proletariado formal, tal como lo sugiere Muntaner et $\mathrm{a}^{36}$ para países de Latinoamérica. La evidencia de estudios con este enfoque es escasa y limitada en América Latina, por lo que este estudio complementa la literatura existente de clase social en este contexto. Los modelos de regresión incorporan ajustes que consideran la distribución de los datos caracterizados por la alta 
concentración de valores que indican ausencia de dolor, entendiendo que este fenómeno es esperable en una población de estudio que forma parte del mercado laboral y que no incluiria a quienes abandonaron el trabajo por condiciones de salud más severas.

Dentro de las limitaciones del estudio, la variable resultado no permite medir la gravedad del dolor o la duración, solo identifica si reporta o no dolor en una localización específica. Por otra parte, el diseño del estudio es transversal, lo que no nos permite identificar univocamente la dirección de la asociación, especialmente en lo que respecta a las conductas relacionadas con la salud.

Nuestros hallazgos muestran un efecto independiente de distintos factores relacionados a la declaración de dolor, identificando importantes diferencias e incrementos de los factores entre los distintos grupos etarios. Esta información es relevante particularmente en los trabajadores de edad avanzada, debido a que la tasa de participación para la población de 65 y más años ha ido en aumento en los últimos años, observándose un aumento en 2,4 puntos porcentuales entre el año 2011 y $2013 .{ }^{37}$

Financiamiento: Este proyecto se realizó con el financiamiento del Fondo de Investigación de la Universidad Mayor (FIDUM).

\section{REFERENCIAS}

1. Becker N, Bondegaard TA, Olsen AK, Sjogren P, Bech P, Eriksen J. Pain epidemiology and health related quality of life in chronic non-malignant pain patients referred to a Danish multidisciplinary pain center. Pain. 1997;73(3):393-400.

2. Elliott $A M$, Smith $B H$, Penny KI, Smith WC, Chambers WA. The epidemiology of chronic pain in the community. Lancet .1999;354(9186):1248-1252.

3. Rojas M, Gimeno D, Vargas-Prada S, Benavides FG. [Musculoskeletal pain in Central American workers: results of the first survey on working conditions and health in Central America]. Rev Panam Salud Publica. 2015;38(2):120-128.

4. Santos AC, Bredemeier M, Rosa KF, Amantea VA, Xavier RM. Impact on the Quality of Life of an Educational Program for the Prevention of Work-Related Musculoskeletal Disorders: a randomized controlled trial. BMC Public Health. 2011;11:60.

5. Von KM, Dworkin SF, Le RL Graded chronic pain status: an epidemiologic evaluation. Pain. 1990;40(3):279-291.

6. Bevan S, Quadrello T, McGee R, Mahdon M, Vavrovsky A, Barham L. Fit for work? Musculoskeletal disorders in the European workforce. London: The Work Fundation; 2009.

7. Breivik $H$, Collett $B$, Ventafridda V, Cohen $R$, Gallacher D. Survey of chronic pain in Europe: prevalence, impact on daily life, and treatment. Eur J Pain. 2006 May;10(4):287-333.

8. Institute of Medicine of the National Academies. Relieving Pain in America: A blueprint for Transforming Prevention, Care, Education, and Research. Washington, DC: National Academy Press; 2011.

9. Hoffmeister L, Vidal C. Desigualdades socieconómicas en salud percibida y declaración de dolor en población trabajadora chilena: ENETS 2009-2010. Persona y sociedad. 2012;26(1):67-86.

10. Bernard BP. Musculoskeletal disorders and workplace factors: a critical review of epidemiologic evidence for workrelated musculoskeletal disorders of the neck, upper extremity, and low back. Cincinnati: NIOSH; 1997.

11. Linton SJ. A review of psychological risk factors in back and neck pain. Spine. 2000;25(9):1148-1156.

12. Palmer KT, Reading I, Linaker $C$, Calnan M, Coggon D. Population-based cohort study of incident and persistent arm pain: role of mental health, self-rated health and health beliefs. Pain. 2008;136(1-2):30-37.

13. Macfarlane GJ, Pallewatte N, Paudyal P, Blyth FM, Coggon D, Crombez G, et al. Evaluation of work-related psychosocial factors and regional musculoskeletal pain: results from a EULAR Task Force. Ann Rheum Dis. 2009;68(6):885-891.

14. Shaw WS, Linton SJ, Pransky G. Reducing sickness absence from work due to low back pain: how well do intervention strategies match modifiable risk factors? J Occup Rehabil. 2006;16(4):591-605.

15. Greenspan JD, Craft RM, LeResche L, Arendt-Nielsen L, Berkley KJ, Fillingim RB, et al. Studying sex and gender differences in pain and analgesia: a consensus report. Pain. 2007;132 Suppl 1:S26-S45.

16. Farioli A, Mattioli S, Quaglieri A, Curti S, Violante FS, Coggon D. Musculoskeletal pain in Europe: the role of personal, occupational, and social risk factors. Scand J Work Environ Health. 2014;40(1):36-46.

17. Blyth FM. Chronic pain--is it a public health problem? Pain. 2008;137(3):465466.

18. Poleshuck EL, Green CR. Socioeconomic disadvantage and pain. Pain. 2008;136(3):235-238.
19. Eachus J, Chan P, Pearson N, Propper C, Davey SG. An additional dimension to health inequalities: disease severity and socioeconomic position. J Epidemiol. Community Health 1999;53(10):603-611.

20. Chile. Ministerio de Salud. Encuesta Nacional de Empleo, Trabajo, Salud y Calidad de Vida, Chile 2013. Santiago: MINSAL; 2014.

21. Wright EO. Class counts: Comoparative studies in class analysis. Cambridge: Cambridge University Press; 2000.

22. Muntaner C, Eaton WW, Diala C, Kessler RC, Sorlie PD. Social class, assets, organizational control and the prevalence of common groups of psychiatric disorders. Soc Sci Med.1998;47(12):2043-53.

23. Rocha KB, Muntaner C, González Rodríguez MJ, Baksai PB, Vallebuona C, Borrell $C$, et al. Social class, health inequalities, and health-related behaviors of working people in Chile. Rev Panam Salud Pública. 2013;33(5):340-348.

24. Orpinas $P$, Valdés $M$, Pemjeam $A$, Florenzano $R$, Nogueira $R$, Hernández J. Validación de una escala breve para la detección de beber anormal (EBBA). En: Florenzano R, Horwitz N, Penna M, et al, editores. Temas de Salud Mental y Atención Primaria de Salud. Santiago: C.P.U.; 1991.

25. Paula G. Modelos de Regressao, com apoio computacional. Instituto de Matemática e estadística. Sao Paulo: Universidade de Sao Paulo; 2004.

26. Greene WH. Accounting for Excess Zeros and Sample Selection in Poisson and Negative Binomial. New York: New York University Stern School of Business; 1994. (NYU Working Paper, EC-94-10).

27. Vuong $\mathrm{OH}$. Likelihood ratio tests for model selection and non-nested hypotheses. Econometrica. 1989;57:303-307.

28. Cameron A, Colin, Trivedi PK. Microeconometrics using stata. College Station: Stata Press; 2009.

29. Reinhardt JD, von EE, Fekete C, Siegrist J. Social inequalities of functioning and perceived health in Switzerland; a representative cross-sectional analysis. PLoS One. 2012;7(6)-e38782:1-7.

30. Artazcoz Lucia, Escribà-Agüir Vicenta, Cortès Imma. Género, trabajos y salud en España. Gac Sanit. 2004;18(2):24-35.

31. Campos-Serna J, Ronda-Pérez E, Artazcoz L, Benavides F. Gender inequalities in occupational health related to the unequal distribution of working and employment conditions: a systematic review. Int J Equity Health. Aug 5 2013;12:57.

32. Chile. Ministerio de Salud. Las inequidades en la salud de los trabajadores y trabajadoras desde una perspectiva de género. Análisis Epidemiológico Avanzado para la Encuesta Nacional de Empleo, Trabajo, Salud y Calidad de Vida de los Trabajadores y Trabajadoras en Chile (ENETS 2009-2010). Santiago: MINSAL; 2011.

33. Punnett L, Wegman DH. Work-related musculoskeletal disorders: the epidemiologic evidence and the debate. J Electromyogr Kinesiol. 2004;14(1):13-23.

34. Muntaner C, Borrell C, Vanroelen C, Chung H, Benach J, Kim IH, et al. Employment relations, social class and health: a review and analysis of conceptual and measurement alternatives. Soc Sci Med. 2010;71(12):2130-2140.

35. van HO, Torrance N, Smith BH. Chronic pain epidemiology-where do lifestyle factors fit in? Brit J Pain. 2013;7(4):209-217.

36. Muntaner C, Rocha KB, Borrell C, Vallebuona C, Ibanez C, Benach J, et al. Social class and health in Latin America. Rev Panam Salud Pública. 2012;31(2):166-75.

37. Chile. Ministerio de Desarrollo Social. Encuesta de Caracterización Socieconómica Nacional 2013. Santiago: Ministerio de Desarrollo Social; 2013. 